\title{
Hipotiroidismo Secundário à Irradiação Cervical: Avaliação Sistemática da Função Tiroideia no Seguimento
}

\author{
Secondary Hypothyroidism after Cervical Irradiation: Systematic Evaluation of \\ Thyroid Function in Follow-up
}

Sara Monteiro GONÇALVES ${ }^{1}$, Brígida da Costa FERREIRA², Maria João GUARDADO ${ }^{1}$, Rui MARQUES ${ }^{1}$, Tânia SERRA ${ }^{1}$, Maria João SERRA ${ }^{1}$, Domingos RODA ${ }^{1}$, Joana BRANDÃO ${ }^{1}$, Gilberto MELO ${ }^{1}$, Maria do Carmo LOPES $^{3}$, Leila KHOURI ${ }^{1}$

Acta Med Port 2014 Jul-Aug;27(4):467-472

\section{RESUMO}

Introdução: A disfunção tiroideia constitui uma consequência, por vezes subestimada, da exposição à radiação. O mecanismo subjacente não está claramente esclarecido, mas terá uma origem multifatorial. Os fatores de risco específicos para o desenvolvimento de hipotiroidismo secundário à radioterapia permanecem indeterminados. A irradiação direta da glândula tiroideia pode resultar, com maior frequência, em hipotiroidismo. Este é uma condição irreversível, requerendo monitorização e tratamento permanente.

Objetivos: Avaliar a incidência de hipotiroidismo nos doentes portadores de neoplasias da cabeça e pescoço submetidos a irradiação cervical, a título intensivo ou adjuvante, bem como determinar se é justificável a integração no protocolo de seguimento Institucional, de parâmetros laboratoriais (TSH, T3 Livre e T4 Livre) para avaliação da função tiroideia neste grupo de doentes e qual a sua periodicidade.

Material e Métodos: Este é um estudo observacional, descritivo, retrospetivo, que engloba um grupo de 376 doentes portadores de neoplasias da cabeça e pescoço, submetidos a tratamento de radioterapia, a título adjuvante ou intensivo, entre os anos de 2007 e 2012, no Instituto Português de Oncologia de Coimbra Francisco Gentil E.P.E. Cumpriram todos os critérios de inclusão 145 doentes. Foram utilizadas escalas de avaliação padronizadas para a definição de hipotiroidismo - LENT-SOMA scales (Late Effects Normal Tissues Subjective Objective Management Analysis). Foi considerada como complicação o hipotiroidismo Grau 1 ou superior.

Resultados: Procedeu-se à análise de um grupo de 145 doentes. A localização tumoral mais frequente foi a Laringe (26,9\%). Trinta e dois doentes efetuaram radioterapia adjuvante e 113 efetuaram esquemas intensivos. A técnica de radioterapia mais utilizada foi a intensidade modulada (IMRT), efetuada em $86,2 \%$ doentes. A taxa de incidência global de hipotiroidismo aos 12 meses foi de $18,6 \%$. Em 51,9\% dos doentes foi efetuado o diagnóstico de hipotiroidismo entre os 9 e os 12 meses após o tratamento de radioterapia.

Discussão: Da análise da literatura publicada, o hipotiroidismo constitui a complicação tiroideia radioinduzida mais frequente e afeta uma grande percentagem de doentes submetidos a radioterapia cervical com intenção curativa. Foi demonstrado que o risco de hipotiroidismo aumenta com o tempo após a exposição, contudo a incidência mais elevada parece ser nos dois primeiros anos após o tratamento. Da nossa análise obtivemos, aos 12 meses uma taxa de incidência global de hipotiroidismo de 18,6\%, (grau 1 de 16,55\% e grau 2 de $2,1 \%$ ).

Conclusão: Neste estudo foi encontrada uma incidência de hipotiroidismo correspondente aos valores mais baixos descritos em outros estudos publicados. O curto tempo de follow-up considerado nesta análise poderá justificar os resultados obtidos. Face aos resultados obtidos, e correlacionando-os com a literatura disponível, os autores consideram justificável e recomendável a inserção da avaliação sistemática da função tiroideia no protocolo de follow-up destes doentes.

Palavras-chave: Neoplasias da Cabeça e Pescoço; Radioterapia; Hipotiroidismo; Estudos de Seguimento.

\section{ABSTRACT}

Introduction: Sometimes Thyroid dysfunction is an underestimated consequence of radiation exposure. The underlying mechanism is not clearly understood, but it is likely to be multifactorial. As so, the specific risk factors associated with the development of secondary radiotherapy hypothyroidism remain undefined. The direct irradiation of the thyroid gland may result more frequently in hypothyroidism. This is an irreversible condition, requiring lifelong treatment and monitoring.

Objectives: To evaluate the incidence of hypothyroidism in patients with head and neck cancer who underwent neck irradiation on an intensive or adjuvant basis and determine whether it is justifiable to integrate the monitoring of parameters for evaluation of thyroid function (TSH, free fraction T3 e free fraction T4 ) in this patient group on the Institutional follow up protocol.

Material and Methods: This is an observational, retrospective descriptive study, which comprises a group of 376 patients with head and neck cancer undergoing radiotherapy, on adjuvant or intensive basis, between the years 2007 and 2012, at Portuguese Institute of Oncology of Coimbra, E.P.E. 145 patients met all the inclusion criteria. Standardized rating scales for the definition of hypothyroidism have been used - LENT-SOMA scales (Late Effects Normal Tissues Subjective Objective Management Analysis). Grade 1 or higher was considered as a complication hypothyroidism.

Results: The analysis of a cohort of 145 patients was carried out. The most frequent tumor site was the larynx (26.9\%). Thirty-two patients received adjuvant radiotherapy and 113 were treated with intensive schemes. The most used radiotherapy technique was intensity modulated (IMRT), performed in $86.2 \%$ patients. The overall incidence rate of hypothyroidism at 12 months was $18.6 \%$. The diagnosis for hypothyroidism was done between 9 and 12 months after treatment radiotherapy in $51.9 \%$ of the patients with this complication.

Discussion: An analysis of the published literature, hypothyroidism is the most common thyroid complication after irradiation and affects

\footnotetext{
1. Serviço de Radioterapia. Instituto Português de Oncologia de Coimbra. Coimbra. Portugal.

2. Departamento de Física. Universidade de Aveiro. Aveiro. Portugal.

3. Serviço de Física Médica. Instituto Português de Oncologia de Coimbra. Coimbra. Portugal.

Recebido: 26 de Dezembro de 2013 - Aceite: 23 de Junho de 2014 | Copyright @ Ordem dos Médicos 2014
} 
a large percentage of patients undergoing cervical radiotherapy with curative intent. It has been shown that the risk of hypothyroidism increases with time after exposure, but the highest incidence appears to be in the first two years after treatment. From our analysis we obtained at 12 months, a rate of incidence of hypothyroidism of $18.6 \%(16.55 \%$ grade 1 and grade $2,2.1 \%)$.

Conclusions: This study found an incidence of hypothyroidism corresponding to the lowest values reported in other published studies. The short duration of follow-up considered in this analysis, may justify these results. With these results and their correlation with the available literature, the authors consider justified and recommend the inclusion of the systematic evaluation of thyroid function in the follow-up protocol of these patients.

Keywords: Head and Neck Neoplasms; Radiotherapy; Hypothyroidism; Follow-up Studies.

\section{INTRODUÇÃO}

A disfunção tiroideia constitui uma consequência, por vezes subestimada, da exposição à radiação. ${ }^{1,2} \mathrm{O}$ mecanismo subjacente à disfunção tiroideia associada à radioterapia $(\mathrm{RT})$ não está claramente esclarecido, mas terá provavelmente uma origem multifatorial. ${ }^{1} \mathrm{~A}$ disfunção tiroideia pós-RT cervical poderá resultar de lesão vascular, lesão celular direta e lesão da cápsula da glândula. ${ }^{1,2}$ Julga-se também que poderá estar relacionada com mecanismos imunológicos. ${ }^{1-4} \mathrm{~A}$ irradiação direta da glândula tiroideia pode resultar, com maior frequência, em hipotiroidismo ${ }^{1,5}$ Outras complicações podem incluir: tiroidite, Doença de Graves, adenomas, bócio multinodular ou mesmo carcinoma. ${ }^{1,4,5-10}$

O hipotiroidismo é uma situação geralmente irreversível, requerendo monitorização e tratamento permanente. ${ }^{11}$ $O$ resultado de alguns estudos que avaliam a qualidade de vida (HRQoL) reportam uma pior HRQoL nos doentes portadores de hipotiroidismo, clínico e mesmo subclínico. ${ }^{11,12}$ No primeiro grupo (doentes sintomáticos), verifica-se um baixo nível de T4 Livre e um alto nível de TSH, já no segundo grupo, há T4 Livre normal e TSH elevada. ${ }^{5,8,12}$ Estima-se que o risco de progressão de hipotiroidismo subclínico para clínico seja de $2-5 \%$ por ano. ${ }^{12}$

Uma vez que esta alteração da função tiroideia pode desenvolver-se anos após o tratamento de RT, a monitorização a longo prazo neste grupo de doentes é recomendada. ${ }^{13-16}$ Atualmente as recomendações internacionais apontam para uma vigilância da função tiroideia a cada 6-12 meses no seguimento destes doentes. ${ }^{17}$

Os fatores de risco específicos para o desenvolvimento de hipotiroidismo secundário à radiação permanecem indeterminados ${ }^{18}$ Existem vários estudos retrospetivos e alguns prospetivos que demonstram uma associação entre o desenvolvimento de hipotiroidismo e a cirurgia (laringectomia, hemitiroidectomia e/ou esvaziamento cervical bilateral), a radioterapia (dose e volume irradiado) e o tempo pós-tratamento. ${ }^{2,13-15}$

\section{OBJETIVOS}

Avaliar a incidência de hipotiroidismo nos doentes portadores de neoplasias da cabeça e pescoço, submetidos a irradiação cervical, a título intensivo ou adjuvante e determinar se é justificável a integração no protocolo de seguimento institucional de parâmetros laboratoriais (TSH, T3 Livre e T4 Livre) para avaliação da função tiroideia neste grupo de doentes e qual a sua periodicidade. Pretende-se também determinar os factores de risco associados ao desenvolvimento desta condição clínica, estudo este, que se encontra ainda em curso.

\section{MATERIAL E MÉTODOS}

Este é um estudo observacional, descritivo, retrospetivo, que engloba um grupo de 376 doentes portadores de neoplasias da cabeça e pescoço, submetidos a RT, a título adjuvante (pós-operatória) ou intensivo (tratamento radical, associado ou não a quimioterapia), entre os anos de 2007 e 2012, no Instituto Português de Oncologia de Coimbra Francisco Gentil E.P.E (IPOC FG). Foram excluídos do estudo todos os doentes cuja função tiroideia não foi avaliada antes do início do tratamento de RT ou aqueles que apresentavam alterações da mesma, previamente ao início do tratamento. Assim, foram excluídos 221 doentes, permanecendo uma amostra de 145 doentes para análise.

Foram recolhidos dados no processo clínico de cada doente, registados durante as consultas de seguimento de radioterapia, no sistema de registo clínico eletrónico para radioterapia, desenvolvido pelo Departamento de Física da Universidade de Aveiro e Serviço de Física Médica e o Serviço de Radioterapia do IPOCFG, RESPONSE.

Os dados foram posteriormente analisados em Excel ${ }^{\circledR}$ 2007.

Foram registadas as características relativas ao doente, tumor e tratamentos efetuados como idade, sexo, localização tumoral, histologia, estádio TNM, tratamentos efetuados (RT, cirurgia, quimioterapia), técnica de RT utilizada e doses administradas.

Foram utilizadas escalas de avaliação padronizadas para a definição de hipotiroidismo - LENT-SOMA scales (Late Effects Normal Tissues Subjective Objective Management Analysis). ${ }^{19-21}$ De acordo com essas escalas, foi considerada como complicação hipotiroismo Grau 1 ou superior. A todos os doentes foram efectuadas avaliações dos valores de TSH, T3 Livre e T4 Livre antes e após terminus da $\mathrm{RT}$, durante um período de 12 meses.

Tendo exclusivamente como critério de inclusão a disponibilidade de controlo analítico tiroideo até aos 24 meses de follow-up, nos doentes em que não foi efectuado o diagnóstico de hipotiroidismo até aos 12 meses, foi constituído um subgrupo acessório de 66 doentes (amostra de conveniência), não constituindo o total do grupo de 145 doentes.

Foi efectuado o delineamento da glândula tiróide em todos os doentes à data do planeamento ou posteriormente naqueles em que não havia sido delineada previamente. As distribuições de dose planeadas no sistema de planeamento Oncentra foram exportadas e utilizadas para converter a dose para um fraccionamento de 2 Gy utilizando o módulo de investigação do RESPONSE. ${ }^{22}$ As doses também foram corrigidas para o número de frações fornecidas e o tempo total de tratamento. A estatística de dose na tiroide para 
todos os doentes foi então determinada.

\section{RESULTADOS}

Procedeu-se à análise de um grupo de 145 doentes (118 do sexo masculino e 27 do sexo feminino). Relação homemlmulher de 4,4:1. Idade média 55,8 \pm 10,9 anos (idade mínima 15,1 anos e máxima 79,4 anos). As localizações tumorais mais frequentes foram a laringe $(26,9 \%)$, a orofaringe $(22,1 \%)$ e a nasofaringe (16,6\%) (Fig. 1). A histologia mais frequente foi o Carcinoma Espinho Celular $(86,8 \%)$.

Trinta e dois doentes efetuaram RT adjuvante e 113 efetuaram esquemas intensivos: 78 efetuaram esquemas concomitantes de RT e quimioterapia (RT\QT), 11 esquemas sequenciais (QT seguida de RT), 22 esquemas de RT isolada e dois doentes foram submetidos a re-irradiação (um concomitante com QT, outro RT isolada). O esquema de quimioterapia mais utilizado foi a cisplatina, em $80.2 \%$ dos doentes. (Tabela 1)

A técnica de RT mais utilizada foi a intensidade modulada (IMRT), efetuada em $86,2 \%$ dos doentes. Os restantes foram submetidos a RT 3D conformacional. A dose média total prescrita foi de $66 \pm$ 4,5 Gy (mínima 58,2 Gy e máxima 70,6 Gy).

Em todos os doentes foram obtidos os valores de avaliação da função tiroideia até 12 meses (inclusive) após conclusão do tratamento de RT. A taxa de incidência global de hipotiroidismo aos 12 meses foi de 18,6\%, com uma incidência de hipotiroidismo grau 1 de $16,6 \%$ e de hipotiroidismo grau 2 de 2,1\%. Não foram registados níveis mais severos de hipotiroidismo. Em 51,9\% dos doentes foi efetuado o diagnóstico de hipotiroidismo entre os 9 e os 12 meses após o tratamento de RT. (Fig. 2)

Alargando o nosso tempo de follow-up para 24 meses,

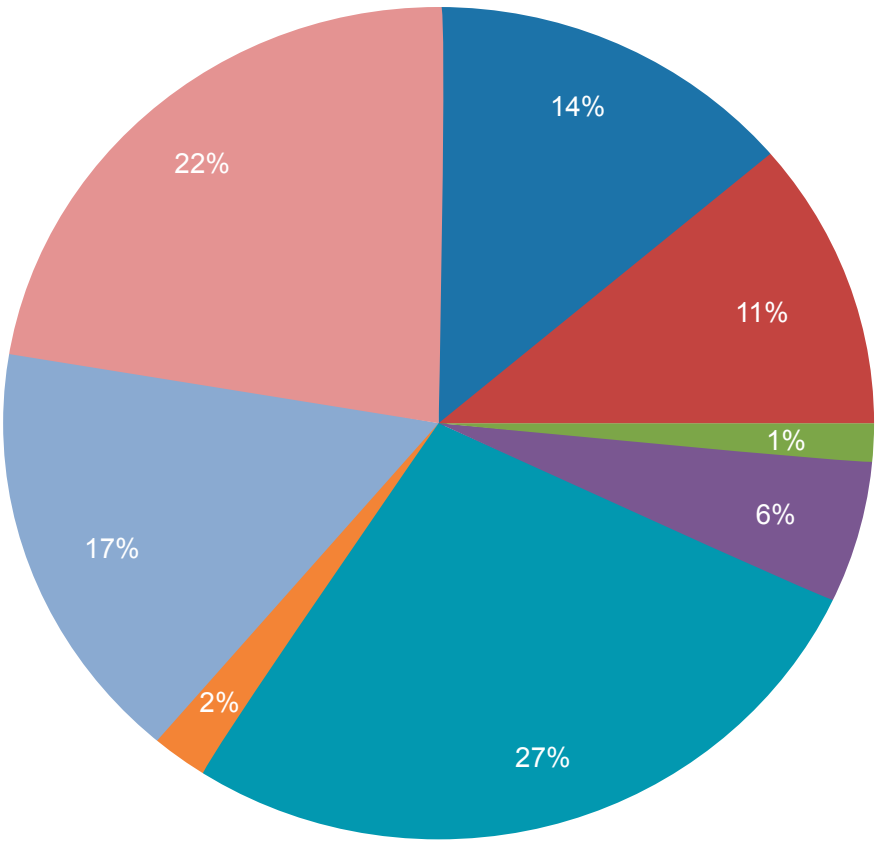

Cav. Oral

Faringo-Laringeo

Gland. Salivar

Hipofaringe

Laringe

Metastases Cervicais

Primário Desconhecido

Nasofaringe

Orofaringe

Figura 1 - Localização Tumoral

Tabela 1 - Esquema de tratamento de RT

Esquema de tratamento de RT

RT
$N$

32

78

11

22

2

145
Re-Irradiação 


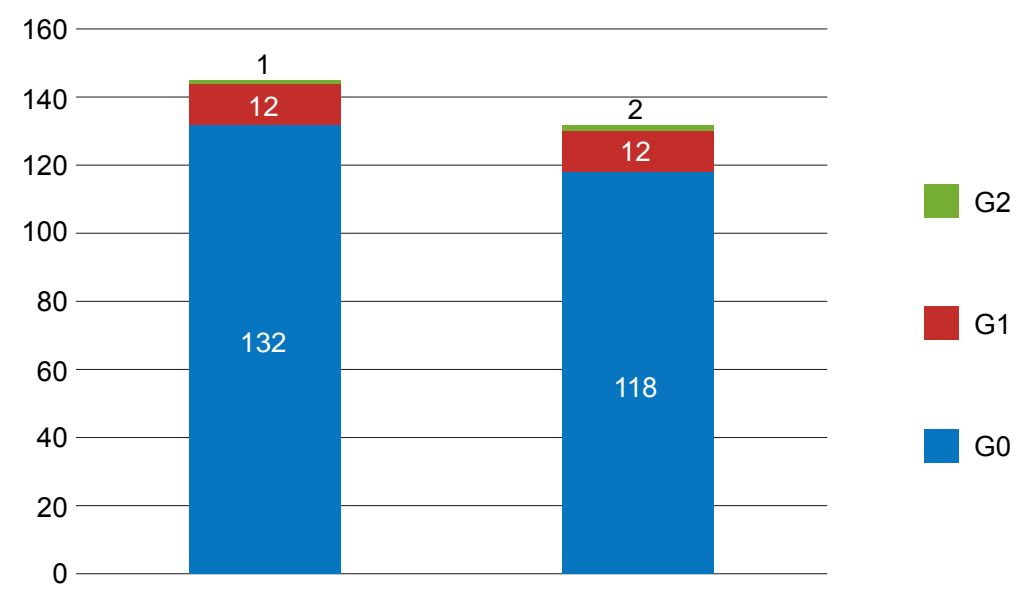

Figura 2 - Incidência de hipotiroidismo para diferentes intervalos de tempo ( $\mathrm{n}^{\circ}$ doentes)

(Utilizadas Escalas de avaliação padronizadas para a definição de hipotiroidismo - LENT-SOMA scales (Late Effects Normal Tissues Subjective Objective Management Analysis) G0, G1 e G2)

66 doentes (45,5\%) do grupo inicial de 145 doentes, sem hipotiroidismo diagnosticado até aos 12 meses, apresentavam registos da função tiroideia. Podemos observar que nesse grupo $27,7 \%$ dos doentes vieram a desenvolver hipotiroidismo entre os 12 e os 24 meses. Hipotiroidismo grau 1 em $21,2 \%$ (14 doentes), grau 2 em 4,5\% (três doentes) e grau 3 em 1,5\% (um doente) (Fig. 2).

A dose média global administrada na glândula tiroideia foi de $44 \pm 16,1 \mathrm{~Gy}$. No grupo de doentes que desenvolveram hipotiroidismo ao longo dos 12 meses de follow-up foi de $48 \pm 7,2 \mathrm{~Gy}$. A dose máxima administrada foi de $58,7 \mathrm{~Gy}$.

\section{DISCUSSÃO}

Muitos dos sintomas de hipotiroidismo, como por exemplo, depressão, letargia, mau estar geral, alterações cutâneas, anedonia, obstipação, entre outros, são frequentemente atribuídos à patologia oncológica de base e em muitas ocasiões são subestimados..$^{23}$

Desde 1961, data em que Feliz et al publicaram o primeiro caso de hipotiroidismo diagnosticado 6 anos após a irradiação cervical de um doente com carcinoma da laringe, já muitas publicações abordaram este tema. ${ }^{23}$

Da análise da literatura publicada, o hipotiroidismo constitui a complicação tiroideia radioinduzida mais frequente $\mathrm{e}$ afeta uma grande percentagem de doentes submetidos a RT cervical com intenção curativa. ${ }^{14,23}$ Com maior frequência, os resultados das várias séries publicadas apontam taxas de incidência de hipotiroidismo que variam entre os $15 \%$ e os $48 \% .{ }^{5}$ No entanto, existem variações descritas desde os $3 \%$ até aos $92 \% .{ }^{5}$ Esta variação dever-se-á provavelmente à patologia de base, história de cirurgia cervical, diferenças no seguimento, pontos de corte analíticos e às distintas técnicas e doses de radiação. ${ }^{1}$ Não existe consenso relativamente à periodicidade de avaliação laboratorial dos doentes em seguimento pós-terapêutica. ${ }^{1,14} \mathrm{~A}$ determinação da função tiroideia neste estudo não foi uniforme no que diz respeito ao período temporal e frequência dos controlos analíticos ao longo dos 12 meses. Este facto constitui uma limitação deste estudo.
Em 2013, Annalisa M. Lo Galbo et al publicaram um estudo prospetivo englobando 137 doentes, portadores de carcinoma da laringe e hipofaringe, num período de follow-up entre os 6 e os 24 meses. ${ }^{13}$ Apresentaram uma alta incidência de hipotiroidismo $(47,4 \%)$, especialmente depois de tratamentos combinados..$^{13} \mathrm{O}$ tempo médio para o desenvolvimento de hipotiroidismo foi de 10 meses..$^{13}$ Recomendam monitorização tiroideia antes do início do tratamento e depois do tratamento: trimestral durante o primeiro ano, semestral durante o segundo ano e anualmente nos anos seguintes. ${ }^{13}$

Foi demonstrado que o risco de hipotiroidismo aumenta com o tempo após a exposição, contudo a incidência mais elevada parece ser nos dois primeiros anos após o tratamento. $1,2,4,23-29$

Da nossa análise obtivemos, aos 12 meses uma taxa de incidência global de hipotiroidismo de 18,6\%, (grau 1 de $16,55 \%$ e grau 2 de $2,1 \%$ ). Alargando o nosso tempo de follow-up para 24 meses, 66 doentes $(45,5 \%)$ do grupo inicial de 145 doentes, sem hipotiroidismo diagnosticado até aos 12 meses, apresentavam ainda registos da função tiroideia. Podemos observar que nesse grupo $27,7 \%$ dos doentes vieram a desenvolver hipotiroidismo entre os 12 e os 24 meses. Hipotiroidismo grau 1 em $21,2 \%$ (14 doentes), grau 2 em 4,5\% (três doentes) e grau 3 em 1,5\% (um doente). Estes resultados são acessórios, não podendo ser considerados representativos por não englobarem a população total dos 145 doentes, tratando-se de uma amostra de conveniência, já que o critério de inclusão foi a disponibilidade de controlo analítico tiroideo até aos 24 meses de follow-up.

Os resultados publicados relativamente à dose administrada e ao volume da glândula irradiada, não são consistentes, mas estes dois fatores parecem influenciar o desenvolvimento de hipotiroidismo. 1,9,26,30,31 Nomeadamente, Bhandare et al, encontraram uma diferença significativa na incidência de hipotiroidismo, quando compararam os doentes que receberam uma dose média na glândula tiroideia superior ou igual a $45 \mathrm{~Gy}$ com aqueles que receberam 
uma dose média inferior a este valor. ${ }^{8}$ No primeiro grupo verificou-se uma incidência significativamente superior de hipotiroidismo ( $27 \%$ aos cinco anos e $39 \%$ aos dez anos), comparativamente aos doentes que receberam uma dose média inferior a 45 Gy (21\% aos cinco e aos dez anos). ${ }^{8}$ Após uma análise preliminar da dose média administrada na glândula tiroideia dos doentes incluídos neste estudo, aos quais foi laboratorialmente documentado hipotiroidismo, conclui-se que esta foi de $48 \pm 7,2$ Gy. Embora a glândula tiroideia não possa ser considerada um órgão limitante de dose, esta deverá ser sempre delineada no planeamento destes doentes.

No caso de doentes portadores de lesões da nasofaringe e em algumas neoplasias da base do crânio, a irradiação da hipófise poderá também contribuir para o desenvolvimento desta alteração tiroideia. ${ }^{1,6,32}$

\section{CONCLUSÕES}

Neste estudo foi encontrada uma incidência de hipotiroidismo correspondente aos valores mais baixos descritos em outros estudos publicados. O curto tempo de follow-up considerado nesta análise poderá justificar estes resultados.

Quando considerados os 24 meses de follow-up e apesar das limitações da amostra, a taxa de incidência aumentou significativamente, pelo que os autores consideram que há necessidade de revisão e continuação deste trabalho no futuro.

Com os resultados obtidos neste estudo e correlacio-

\section{REFERÊNCIAS}

1. Couto J, Santos AP, Torres I. Revisão doença da tiroide secundaria a radioterapia. Rev Port Endocrinol Diabetes Metab. 2013;8:77-81.

2. Lo Galbo AM, de Bree R, Kuik DJ, Lips PT, Mary B, von Blomberg E, et al. The prevalence of hypothyroidism after treatment for laryngeal and hypopharyngeal carcinomas: are autoantibodies of influence? Acta Otolaryngol. 2007;127:312-7.

3. Turner SL, Tiver KW, Boyages SC. Thyroid dysfunction following radiotherapy for head and neck cancer. Int J Radiat Oncol Biol Phys. 1994;31:279-83.

4. Hancock SL, Cox RS, McDougall IR. Thyroid diseases after treatment of Hodgkin's disease. N Eng J Med. 1991;325:599-605.

5. Bakhshandeh M, Hashemi B, Mahdavi SR, Nikoofar A, Vasheghani $M$, Kazemnejad A. Normal tissue complication probability modeling of radiation-induced hypothyroidism after head-and-neckradiation therapy. Int J Radiation Oncol Phys. 2013;85:514-21.

6. Jereczek-Fossa BA, Alterio D, Jassem J, Gibelli B, Tradati N, Orecchia R. Radiotherapy induced thyroid disorders. Cancer Treat Rev. 2004;30:369-84.

7. Alterio D, Jereczek-Fossa BA, Franchi B, D'Onofrio A, Piazzi V, Rondi $E$, et al. Thyroid disorders in patients treated with radiotherapy for head and neck cancer: a retrospective analysis of seventy three patients. Int J Radiat Oncol Biol Phys. 2007;67:144-50.

8. Bhandare N, Kennedy L, Malyapa RS, Morris CG, Mendenhall WM. Primary and central hypothyroidism after radiotherapy for head and neck tumors. Int J Radiat Oncol Biol Phys. 2007;68:1131-9.

9. Kuten A, Lubochitski R, Fishman G, Dale J, Stein ME. Postradiotherapy hypothyroidism: radiation dose response and chemotherapeutic radiosensitization at less than 40 Gy. J Surg Oncol. 1996;61:281-3.

10. Hancock SL, McDougall IR, Constine LS. Thyroid abnormalities after therapeutic external radiation. Int J Radiat Oncol Biol Phys. 1995;30:116570 .

11. Boomsma MJ, Bijl HP, Langendijk JA. Radiation-induced hypothyroidism in head and neck cancer patients: a systematic review. Rad Oncol. nando-os com a literatura disponível, os autores consideram justificável e recomendável a inserção da avaliação sistemática da função tiroideia no protocolo de follow-up destes doentes. Esta deverá ser avaliada, por rotina, antes do início dos tratamentos e durante o follow-up, em intervalos mais curtos nos dois primeiros anos e até um mínimo de cinco anos após conclusão do tratamento. De acordo com as recomendações internacionais e a prática actual dos autores, é recomendável a vigilância da função tiroideia a cada 6-12 meses, no seguimento dos doentes submetidos a irradiação cervical.

Será também recomendável o delineamento da glândula tiroideia no planeamento destes doentes, embora esta não possa ser considerada um órgão limitante de dose. Permitirá determinar o volume de glândula irradiado e a dose recebida e correlacionar estes fatores com a clínica apresentada em follow-up. Por último, outra vantagem no delineamento deste órgão, será uma melhor otimização dos planeamentos de IMRT que, ao utilizar a técnica de planeamento inverso, irá permitir minimizar a dose recebida pela glândula.

\section{CONFLITO DE INTERESSES}

Os autores declaram não ter nenhum conflito de interesses relativamente ao presente artigo.

\section{FONTES DE FINANCIAMENTO}

Fundação para Ciência e Tecnologia com o programa PEst-C/CTM/LA0025/2011.

12. Vigário $P$, Teixeira $P$, Reuters $V$, Almeida $C$, Maia M, Silva M, et al. Perceived health status of women with overt and subclinical hypothyroidism. Med Princ Pract. 2009;18:317-22.

13. Lo Galbo AM, Kuik DJ, Lips P, von Blomberg BM, Bloemena E, Leemans $C R$, et al. A prospective longitudinal study on endocrine dysfunction following treatment of laryngeal or hypopharyngeal carcinoma. Oral Oncol. 2013;49:950-5.

14. Garcia-Serra A, Amdur RJ, Morris CG, Mazzaferri E, Mendenhall WM. Thyroid function should be monitored following radiotherapy to the low neck. Am J Clin Oncol. 2005;28:255-8.

15. Turgut OK, Erişen L, Coşkun H, Basut O, Onart S, Hizalan I. Hypothyroidism after primary surgical treatment for laryngeal and hypopharyngeal cancer. Kulak burun bogaz intis derg. 2008;18:125-30.

16. Tell R, Lundell G, Nilsson B, Sjödin H, Lewin F, Lewensohn R. Long-term incidence of hypothyroidism after radiotherapy in patients with head-and-neck cancer. Int J Radiat Incol Biol Phys. 2004;60:395-400.

17. NCCN Guidelines - Head and Neck Cancers Version 1. 2014.

18. Ramos CM, Urdaneta Al, Wan W, Chang MG, Song S. Bilateral neck irradiation confers a higher risk of developing hypothyroidism in patients with head-and-neck cancer. Rev Port Endocrinol Diabetes Metab. 2013;8:77-81.

19. Pavy JJ, Denekamp J, Letschert J, Littbrand B, Mornex F, Bernier J, et al. EORTC Late Effects Working Group. Late effects toxicity scoring: The SOMA scale. Radiother Oncol. 1995;35:11-15.

20. Rubin P, Constine LS 3rd, Fajardo LF, Phillips TL, Wasserman TH. Overview of late effects normal tissues (LENT) scoring system. Radiother Oncol. 1995;35:9-10.

21. Ho KF, Farnell DJ, Routledge JA, Burns MP, Sykes AJ, Slevin NJ, et al. Comparison of patient-reported late treatment toxicity (LENT-SOMA) with quality of life (EORTC QLQ-C30 and QLQ-H\&amp; N35) assessment after head and neck radiotherapy. Radiother Oncol. 2010;97:2705. 
22. Ferreira $B C$, do Carmo Lopes $M$, Mateus $\mathrm{J}$, Capela $M$, Mavroidis $P$. Radiobiological evaluation of forward and inverse IMRT using different fractionations for head and neck tumours. Radiat Oncology. 2010;5:57.

23. Mercado G, Adelstein DJ, Saxton JP, Secic M, Larto MA, Lavertu P. Hypothyroidism: A frequent event after radiotherapy and after radiotherapy with chemotherapy for patients with head and neck carcinoma. Cancer. 2001;92:2892-7.

24. Colevas AD, Read R, Thornhill J, Adak S, Tishler R, Busse P, et al. Hypothyroidism after multimodality treatment for stage III and IV squamous cell carcinomas of the head and neck. Int $\mathrm{J}$ Radiation Oncology Biol Phys. 2001;51:599-604.

25. Sklar C, Whitton J, Mertens A, Stovall M, Green D, Marina N, et al. Abnormalities of the thyroid in survivors of Hodgkin's disease: Data from the Childhood Cancer Survivor Study. J Clin Endocrinol Metab. 2000;85:3227-32.

26. Gal RL, Gal TJ, Klotch DW, Cantor AB. Risk factors associated with hypothyroidism after laryngectomy. Otolaryngol Head Neck Surg. 2000;123:211-7.

27. Nishiyama K, Tanaka E, Tarui Y, Miyauchi K, Okagawa K. A prospective analysis of subacute thyroid dysfunction after neck irradiation. Int J Radiat Oncol Biol Phys. 1996;34:439-44.

28. Aich RK, Ranjan DA, Pal S, Naha BL, Amitabh R. latrogenc hypothyroidism: a consequence of external beam radiotherapy to the head $\&$ neck malignancies. J Cancer Res Ther. 2005;1:142-6.

29. Tell R, Sjödin H, Lundell G, Lewin F, Lewensohn R. Hypothyroidism after external radiotherapy for head and neck cancer. Int J Radiat Oncol Biol Phys. 1997;39:303-8.

30. Lin Z, Wu VW, Lin J, Feng H, Chen L. A longitudinal study on the radiation-induced thyroid gland changes after external beam radiotherapy of nasopharyngeal carcinoma. Thyroid. 2011;21:19-23.

31. Diaz R, Jaboin JJ, Morales-Paliza M, Koehler E, Phillips JG, Stinson S, et al. Hypothyroidism as a consequence of intensity-modulated radiotherapy with concurrent taxane-based chemotherapy for locally advanced head-and-neck cancer. Int J Radiat Oncol Biol Phys. 2010;77:468-76.

32. Vanderpump MP, Tunbridge WM, French JM, Appleton D, Bates D, Clark $\mathrm{F}$, et al. The incidence of thyroid disorders in the community: a twenty-year follow-up of the Whickham Survey. Clin Endocrinol. 1995;43:5568. 
Sara Monteiro GONÇALVES, Brígida da Costa FERREIRA, Maria João GUARDADO, Rui MARQUES, Tânia SERRA, Maria João SERRA, Domingos RODA, Joana BRANDÃO, Gilberto MELO, Maria do Carmo LOPES, Leila KHOURI

\section{Hipotiroidismo Secundário à Irradiação Cervical: Avaliação Sistemática da Função Tiroideia no Seguimento \\ Acta Med Port 2014:27:467-472}

Publicado pela Acta Médica Portuguesa, a Revista Científica da Ordem dos Médicos

Av. Almirante Gago Coutinho, 151

1749-084 Lisboa, Portugal.

Tel: +351 218428215

E-mail: submissao@actamedicaportuguesa.com

www.actamedicaportuguesa.com

ISSN:0870-399X | e-ISSN: 1646-0758

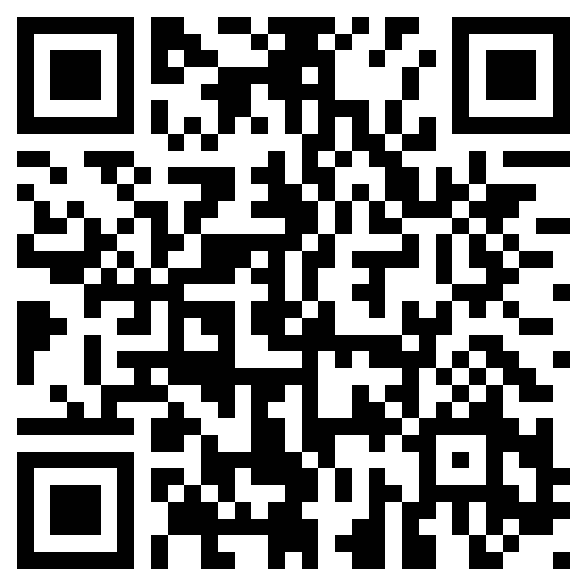

